EPJ Web of Conferences 20, 04002 (2012)

DOI: $10.1051 /$ epjconf/20122004002

(C) Owned by the authors, published by EDP Sciences, 2012

\title{
Formation and Evolution of Neutron Star Binaries : Masses of Neutron Stars
}

\author{
Chang-Hwan Lee \\ Department of Physics, Pusan National University, Pusan 609-735, Korea
}

\begin{abstract}
Neutron star (NS) is one of the most interesting astrophysical compact objects for hardronic physics. It is believed that the central density of NS can reach several times the normal nuclear matter density $\left(\rho_{0}\right)$. Hence, the inner part of NS is the ultimate testing place for the physics of dense matter. Recently, the mass of NS in a NS-white dwarf (WD) binary PSR J1614-2230 has been estimated to be $1.97 \pm 0.04 M_{\odot}$ [1]. Since this estimate is based on the observed Shapiro delay, it can give the lower limit of the maximum NS mass and rules out many soft equations of state. On the other hand, all the well-measured NS masses in NS-NS binaries are smaller than $1.5 M_{\odot}$. In this work, by introducing the supercritical accretion during the binary evolution, we propose a possibility of forming higher mass NS in NS-WD binaries. In this scenario, the lifetimes of NS and WD progenitors are significantly different, and NS in NS-WD binary can accrete $>0.5 M_{\odot}$ after NS formation during the giant phase of the progenitor of WD. On the other hand, for the binary system with NS and heavier $\left(>8 M_{\odot}\right)$ giants, the first-born NS will accrete more from the companion and can collapse into black hole. The only way to avoid the supercritical accretion is that the initial masses of progenitors of NS binary should be very close so that they evolve almost at the same time and don't have time to accrete after NS formation.
\end{abstract}

\section{Introduction}

Dense hadronic matter is the system in which the densities are higher than normal nuclear matter density $\rho_{0}$. Both theoretical approaches and heavy ion experiments indicate that the hadronic properties, such as mass, decay constants, and interaction couplings, etc, can change dramatically in dense hadronic matter. Many NS equation of states (EOS) with medium dependent hadronic properties have been calculated but is's still quite uncertain which EOS is correct. Since, inside of NS cannot be observed directly, finding the maximum mass of NS is one of the most important questions to be answered.

Recent discovery of $1.97 M_{\odot}$ NS, PSR J1614-2230, in a NS-WD binary has been reported [1]. This observation is very important because this mass measurement is based on the Shapiro delay, for the first time in NS-WD binaries. So, NRAO announced that this is the first observation of massive $\left(\geq 2 M_{\odot}\right)$ neutron star by mentioning "Astronomers Discover Most Massive Neutron Star Yet Known" in their homepage[2]. However, neutron stars in various binaries are summarized in Lattimer and Prakash [3], and there are several neutron stars with masses heavier than $2 M_{\odot}$. One may wonder why NRAO announced that they discovered the most massive neutron star in 2010, neglecting those heavy neutron stars reported earlier in the literature [3]. In this review, I would like to discuss the importance of the discovery of J1614-2230 and the uncertainties in the measurement of previous massive neutron stars. If the mass of NS in PSR J1614-2230 is finally confirmed to be $1.97 M_{\odot}$, the maximum mass of NS has to be bigger than $\sim 2 M_{\odot}$. This will rule out many EOS and can give constraints on many hadronic properties beyond normal nuclear matter density.

\footnotetext{
${ }^{a}$ e-mail: clee@pusan.ac.kr
}

This is an Open Access article distributed under the terms of the Creative Commons Attribution-Noncommercial License 3.0, which permits unrestricted use, distribution, and reproduction in any noncommercial medium, provided the original work is properly cited. 


\section{EPJ Web of Conferences}

On the other hand, all well-measured NSs in double NS binaries [3] have masses $<1.5 M_{\odot}$. In the previous work [4], Lee et al. tried to explain this observation by introducing supercritical accretion in the evolution process of NS-NS binaries. In this work[4], they argued that the NS masses in NS-NS binaries cannot be the indication of the maximum NS mass unless one understands the formation and evolution history of neutron star binaries. The main conclusion was that there can exist a gap, due to the supercritical accretion, in the NS mass distribution in NS binaries and the maximum NS mass can be higher than those observed in NS-NS binaries. In order to understand the NS masses in NS binaries, one has to understand the formation and evolution history of NS binaries.

In this review, we will discuss the role of supercritical accretion in the formation and evolution of NS binaries, and will argue that the $1.97 M_{\odot}$ NS in a NS-WD binary might be the result of the supercritical accretion[5]. The lack of supercritical accretion in NS-NS binaries can explain the current observation that all well measured NS masses in NS-NS binaries are $<1.5 M_{\odot}$. Hence, this limit is independent of the maximum mass of NS, but the result of evolution of NS binaries. We discuss that NS-NS binaries are formed when the initial mass difference of two progenitors of double neutron stars is less than $4 \%$ so that they don't have enough time to accrete after neutron stars are formed. When the initial mass difference is bigger than $4 \%$, the the first born NS can accrete during the giant phase of the companion. Obviously, the progenitors of white dwarf are $<8 M_{\odot}$ and the mass differences from the NS progenitors are usually bigger than $4 \%$. So, we suggest that the supercritical accretion to the first-born NS can explain both high mass NS in NS-WD binaries and the non-existence of high mass NS in NS-NS binaries.

\section{Formation and evolution of NS binaries}

In order to understand the mass of NSs in various NS binaries, the differences and similarities in the formation and evolution of binaries have to be considered. In this section, we will discuss the possibility of supercritical accretion onto NS during the giant phase of the companion and their consequences to the final mass of NS in various binaries.

\subsection{Eddington limit and supercritical accretion}

Eddington limit is the condition that the photon pressure balances the gravitational force. Eddington luminosity, which satisfies this balance in the spherical accretion, is given as

$$
L_{\text {Edd }}=\frac{4 \pi c G M}{\kappa} \approx 1.3 \times 10^{38} \frac{M}{M_{\odot}} \mathrm{erg} \mathrm{s}^{-1} .
$$

Here $M$ is the mass of the accreting star and $\kappa=\sigma_{T} N_{A}$ with Thomson scattering cross section $\sigma_{T}$ and Avogadro's number $N_{A}$ [6]. This luminosity can be related to the mass accretion rate with some efficiency $\eta$

$$
L_{\mathrm{Edd}}=\eta \dot{M}_{\mathrm{Edd}} c^{2} .
$$

Hence the Eddington mass accretion rate can be written as

$$
\dot{M}_{\mathrm{Edd}}=\frac{4 \pi G M}{\kappa c \eta} \approx 0.45 \times 10^{-8}\left(\frac{M}{M_{\odot}}\right) M_{\odot} \mathrm{yr}^{-1} .
$$

When the accretion rate is $<10^{3} \dot{M}_{\text {Edd }}$, the star cannot accrete more than the Eddington limit with some uncertainty in determining the efficiency.

When the accretion rate is $>10^{3} \dot{M}_{\text {Edd }}$, Brown and Weingartner [7] discussed the possibility of supercritical accretion. The main reason is the thermal neutrino pair production. When the accretion rate is large enough, the temperature can reach the point in which neutrino pair production wins the photon production. Since neutrinos are weakly interacting, the generated neutrino pairs can leave the system without interaction, i.e., almost no contribution to the pressure. Whether supercritical accretion can appears or not is still quite uncertain. But, in this review, we assume that the supercritical accretion happens during the binary evolution of NS binaries. 


\subsection{The fate of first-born NS star in NS-NS binaries}

As summarized in Lattimer and Prakash [3], all well measured neutron star masses in double neutron star binaries have masses $<1.5 M_{\odot}$. In this section, we discuss the formation and evolution of NS-NS binaries.

The massive progenitors of NS binaries with initial masses $M_{\text {ZAMS }}$ have to pass the giant phase during the evolution in which the stars expand. Here $M_{\text {ZAMS }}$ is the initial mass of main sequence stars when they form, and ZAMS indicatess zero age main sequence. Massive Stars with $M_{\mathrm{ZAMS}}>8 M_{\odot}$ can produce NS in the core at the end of their lifetime. In order to form the close binaries which we observe today, the progenitors of NS and the companion have to be in a certain range in distance, so that they can come close during the giant phase of NS progenitors. Since the lifetimes of the progenitors are inversely proportional to their masses, $\tau \propto 1 / M_{\text {ZAMS }}^{2.5}$, the more massive progenitor produces NS first. We call this first-born NS as primary NS.

Numerical simulation of giant stars in binaries [8] indicates that the iron core masses never grow beyond $1.5 M_{\odot}$. Since NS is formed by the collapse of iron core, one can assume that the fresh NS mass to be $<1.5 M_{\odot}$ which is independent of NS EOS. This indicates that the well measured masses in NS-NS binaries might be the result of binary evolution independently of NS EOS. In this review, we assume that the fresh NS mass is $<1.5 M_{\odot}$.

One can consider two difference cases below.

\subsubsection{Case I: ZAMS mass difference is bigger than $4 \%$.}

When ZAMS mass difference is bigger than $4 \%$, the difference in their lifetime is bigger than $10 \%$. Progenitors of NSs spend $90 \%$ of their lifetime in main sequence. So, in this case, when the primary NS is formed, the companion is still in main sequence. When the companion star expand in its giant phase, if the primary NS is within the radius of the expanding giants, the NS will go into the envelope of expanding giant. In this stage, the NS can accrete significant amount of mass from the companion. How much mass will be accreted is quite uncertain and strongly depends on a few coefficient which controls the amount of accretion[9]. Firstly, one has to consider the energy loss rate due to the accretion on NS with mass $M_{\mathrm{NS}}$,

$$
\dot{E}_{\mathrm{acc}}=\frac{1}{2} c_{d} \frac{G\left(M_{\mathrm{NS}}+M_{\text {giant }}\right)}{a} \dot{M}_{\mathrm{NS}}
$$

where $M_{\text {giant }}$ is the mass of giant (donor), $a$ is the binary separation, and $c_{d}$ is the drag coefficient which controls the energy loss rate due to the accretion on the compact star. The orbital energy is dissipated due to the dynamical friction of accreting NS, hence one can equate $\dot{E}_{\text {acc }}=-\dot{E}_{\text {orb }}[9,10]$, where

$$
-\dot{E}_{\mathrm{orb}}=\frac{1}{2} \frac{G M_{\text {giant }}}{a} \dot{M}_{\mathrm{NS}}+\frac{1}{2} \frac{G M_{\mathrm{NS}}}{a} \dot{M}_{\text {giant }}-\frac{1}{2} \frac{G M_{\mathrm{NS}} M_{\text {giant }}}{a^{2}} \dot{a} .
$$

Secondly, during the accretion phase the binding energy of the giant envelope can be written as[10]

$$
E_{\text {bind }}=-\frac{1}{\lambda} \frac{G M_{\text {giant }}\left(M_{\text {giant }}-M_{\text {giant,core }}\right)}{a}
$$

where $\lambda$ is the numerical factor which is related with the structure of giant envelope. Finally, these two energies are related during the accretion phase $[9,10]$

$$
\alpha_{\mathrm{ce}} \frac{d E_{\mathrm{orb}}}{d M_{\text {giant }}}=-\frac{d E_{\mathrm{bind}}}{d M_{c}} .
$$

Hence the mass accretion depends on three coefficients $c_{d}, \lambda$ and $\alpha_{\mathrm{ce}}$. However, since the equation depends on the combination $\alpha_{\mathrm{ce}} \lambda$, two effective paparmeters $c_{d}$ and $\alpha_{\mathrm{ce}} \lambda$ control the accretion. Lee et al.[4] used $c_{d}=6$ [11] and $\alpha_{\mathrm{ce}} \lambda=1.0$. Using these parameters, Lee et al. [4] estimated the mass accretion to be $>0.3 M_{\odot}$. Recently, Lee [5] found that the mass accretion can be $>0.5 M_{\odot}$ with different parameters, $\alpha_{\mathrm{ce}} \lambda=0.2$, which are fixed by the evolution of soft X-ray binaries [12]. 


\section{EPJ Web of Conferences}

\subsubsection{Case II: ZAMS mass difference is less than $4 \%$.}

If the ZAMS mass difference is less than $4 \%$, the difference in the lifetime of two progenitors is less than $10 \%$, which means that when the progenitor of the first NS is expanding in giant phase, the companion is also in expanding phase. In this case, the binary goes into common envelope evolution because the expanding envelope overlap. When the first NS is formed, the companion already lost significant amount of hydrogen envelope and there is not enough time and envelope left for the supercritical accretion. As a result, both NSs are similar in mass, and form the NS-NS binary in which the masses of both NSs are $<1.5 M_{\odot}$ consistent with current observation[3]. One can check from the observed data [3] that NS masses in NS-NS binaries are close in mass; the difference is less than $\sim 5 \%$. Note that there can be an additional mass accretion during the super-giant (He-shell burning) phase of the companion unless the progenitors are almost the same in mass, usually the time in super-giant phase is $O(1 \%)$ of the total lifetime.

\subsection{Maximum mass of NS from evolutionary point of view}

In the discussion above, we argued that the first-born NS can accrete significant amount mass when the ZAMS mass difference of their progenitors is $>\sim 5 \%$. On the other hand, when the mass difference is less than $\sim 4 \%$, the first-born NS has no time for the supercritical accretion and remain as $<1.5 M_{\odot}$.

Hence, there can be a gap in the mass distribution of NSs in NS-NS binaries if the first-born NS remains as NS. Lee et al. [4] argued that the probability of having supercritical accretion (having the ZAMS mass difference of their progenitors $>4 \%$ ) is significantly larger than the probability of having mass difference $<4 \%$. In this scenario, probability of having high mass NS $\left(>1.8 M_{\odot}\right)$ is a few times larger than the probability of having only low mass $\left(<1.5 M_{\odot}\right)$ NS. However, all well-measured NS masses in NS-NS binaries are below $1.5 M_{\odot}$. Based on this observation and the supercritical accretion, Lee et al. argued that the first-born NS with significant amount of mass accretion due to the supercritical accretion went into a black hole. In this case, the maximum NS mass can be higher than $1.5 M_{\odot}$ independently of the observed NS masses in NS-NS binaries.

Of course, due to the uncertainty in the parameters, $c_{d}, \alpha_{\mathrm{ce}}, \lambda$, during the evolution one cannot pin down the upper limit of possible range in which the maximum NS mass can lie. As we will discuss in the next section, however, by assuming that maximum NS mass is $>2 M_{\odot}$, we found that the supercritical accretion at least can explain both 1) the high mass NS in NS-WD binaries, and 2) the non-existence of high mass NS in NS-NS binaries.

\subsection{Formation of Massive NS in NS-WD binaries}

Recently discovery of $2 M_{\odot}$ NS, PSR J1614-2230, in NS-WD has been reported [1]. This observation is very important because this mass measurement is based on the Shapiro delay for the first time in NS-WD binaries. NRAO announced that this is the first observation of massive $\left(\geq 2 M_{\odot}\right)$ neutron star by mentioning “Astronomers Discover Most Massive Neutron Star Yet Known”. In Lattimer and Prakash [3], one can find a few high mass $\left(>2 M_{\odot}\right)$ NSs in NS-WD binaries. However, since they are based on Bayesian analysis, not on the Shapiro delay observation, the uncertainties involved in the mass estimation is very large. One example is J0751+1807. NS mass in J0751+1807 was reported to be $2.1 \pm 0.2 M_{\odot}$ [13], however, it turned out to be $1.26_{-0.12}^{+0.14} M_{\odot}$ later[14]. This is the reason why astronomers announced that $\mathbf{J} 1614-2230$ is the discovery of most massive NS yet known.

High mass $\left(>2 M_{\odot}\right)$ NSs are also reported in X-ray pulsar [3]. However, since the observers didn't consider them as the evidence of the existence of high mass NS, one has to consider the uncertainties involved in the mass estimation in X-ray pulsars. In X-ray pulsar binary, the companion of NS is $\mathrm{He}$ star which is not a compact star. During the orbital motion, NS evan can go into the envelope of He star. The main question is what is the real center of mass of the companion He star. Since one cannot directly observe the interior of He star, one cannot really pin down the NS mass in X-ray pulsar case. There are many recent works that discuss the uncertainties in the mass estimation in X-ray pulsars. Recently, 
Steiner et al. [15] showed the NS mass strongly depend on the assumption of the photosphere. Güver et al.[16,17] showed that one can even get two pairs of values in X-ray pulsars.

Considering the uncertainties in previous mass estimates in NS-WD and X-ray pulsar, the recent discovery of $2 M_{\odot}$ NS, PSR J1614-2230, is very important because it is based on the Shapiro delay which estimates the time delay of pulsar signal when they passes the gravitational field of WD.

Now the question is how one can understand the formation of high mass $\left(\sim 2 M_{\odot}\right)$ NS in NS-WD binaries while no such high mass NS has been found in NS-NS binaries.

The ZAMS mass difference of progenitors of NS-WD binaries are usually $>4 \%$. This indicates that the supercritical accretion can happen. Since the ZAMS mass of WD progenitors are $<8 M_{\odot}$, the total amount of accretion will be smaller than NS-NS or NS-BH binaries considered in the previous section. Hence the NS masses in NS-WD binaries can be in a wide range; $1.1 M_{\odot} \sim 2.0 M_{\odot}$ while NS masses in NS-NS binaries are $>\sim 2 M_{\odot}$ if the first-born NS remains as NS. Lee et al.[4] claimed that the first-born NS in NS-NS binaries went into BH due to the supercritical accretion, finally forming NS-BH binaries. Of course, both the lower and upper limits are not known well.

\section{Prospects for Other Astrophysical Phenomena}

In this review, we discussed the implication of supercritical accretion onto the first-born NS in NS binaries. The main concern in this review is the mass of NS that can be observed.

On the other hand, there are many other developments in both hadronic and astrophysics in connection with NS.

In hadronic side, due to the recent observation of J1614-2230, many NS equation of states will be ruled out once the NS mass $1.97 M_{\odot}$ is confirmed. Since many equation of states with exotic states/particles give lower maximum NS mass, one has to reconsider the role of exotic states and particles inside NS. One example is the kaon condensation and strange quark star. Since nucleons are composed of up and down quarks only, it's quite natural for strangeness to come in and the strangeness will reduce the maximum mass of NS. There are many new theoretical developments that are based on the symmetries, such as AdS/QCD. We believe that such a new approached that are based on the fundamental symmetries will give clues in understanding the properties of NS.

In astrophysical side, there are many recent developments on gamma-ray bursts (GRB) and gravitational wave radiation. There are two classes of GRBs, long-duration GRB ( $\tau>2 \mathrm{sec})$ and short-hard GRB (SHB, $\tau<2 \mathrm{sec}$ ) which are classified according to their lifetime $\tau$. One of the main sources of SHBs is believed to be NS binaries. When the two compact stars in NS binaries merge, strong gammaray can be generated. At the same time, since these are the mergers of very compact objects, strong gravitational wave will be radiated. During the merger, due to the strong gravity, NS may break up and reveal some inner properties. So, in the near future, we may be able to learn hadronic physics (inside of NS) from GRBs and gravitational wave radiation.

\section{Acknowledgment}

We would like to thank APCTP where this work has been presented and discussed during the International Workshop on Hyper Nuclear Physics 2011 which was held at the APCTP headquarter. This work is supported for two years by Pusan National University Research Grant.

\section{References}

1. P.B. Demorest, T. Pennucci, S.M. Ransom, M.S.E. Roberts, and J.W.T. Hessels, Nature 467, (2010) 1081.

2. The National Radio Astronomy Observatory homepage, http://www.nrao.edu/pr/2010/bigns/

3. J. Lattimer and M. Prakash, Physics Reports 442, (2007) 109.

4. C-H. Lee, H.-J. Park, and G.E. Brown, Astrophysical Journal 670, (2007) 741. 
5. C.-H. Lee, in preparation (2011).

6. C.-H. Lee, Journal of the Korean Physical Society 49, (2006) 1791

7. G.E. Brown and J.C. Weingartner, Astrophysical Journal 436, (1994) 843

8. G.E. Brown, A. Heger, N. Langer, C.-H. Lee, S. Wellstein, H.A. Bethe, New Astronomy 6, (2001) 457

9. H. Bethe and G.E. Brown, Astrophysical Journal 506, (1998) 780

10. K. Belczynski, V. Kalogera, and T. Bulik, Astrophysical journal 572, (2002) 407

11. E. Shima, T. Matsuda, H. Takeda, and K. Sawada, Monthly Notices of the Royal Astronomical Society 217, (1985) 367

12. C.H. Lee, G.E. Brown, and R.A.M.J. Wijers, Astrophysical Journal 575, (2002) 996

13. D.J. Nice et al. Astrophysical Journal 634, (2005) 1242

14. D.J. Nice, tlak at the conference "40 Years of Pulsar", Aug. 12-17, 2007, McGill, Canada

15. A.W. Steiner, J.M. Lattimer, E.F. Brown, arXiv:1005.0811

16. T.Güver, D. Psaltis, and F. Özel, arXiv:1103.5767

17. T. Güver, F. Özel, and D. Psaltis, arXiv:1104.2602 